\title{
Multiregion segmentation of microcalcification in mammogram images by using Parametric Kernel Graph Cut algorithm
}

\author{
A. A Malek ${ }^{1,}$, N. A Rahim ${ }^{1}$, N.F.N Mushtafa ${ }^{1}$, N.A. Zailan ${ }^{1}$ and N. Mohamed ${ }^{1}$ \\ 1 Faculty of Computer and Mathematical Sciences, Universiti Teknologi MARA Cawangan Negeri Sembilan, 70300 Seremban, Negeri Sembilan, Malaysia
}

ABSTRACT - Early detection of breast cancer can be detected through screening mammography. However, the potential abnormality such as microcalcification can hardly be differentiated by the radiologists due to the tiny size, which sometimes be hidden behind the density of breast tissue. Therefore, image segmentation technique is required. This paper proposes the potential use of Parametric Kernel Graph Cut Algorithm in segmenting microcalcification. The performances of this method were measured based on accuracy, sensitivity, Dice and Jaccard coefficient. All the experimental results generated satisfying results, whereby all images produced the average of $91.67 \%$ for Dice coefficient and $84.72 \%$ for Jaccard coefficient. Meanwhile, both accuracy and sensitivity results acquired $97.84 \%$ and $96 \%$, respectively. Therefore, Parametric Kernel Graph Cut algorithm had proved its ability to segment the microcalcification robustly and efficiently.

\author{
ARTICLE HISTORY \\ Revised: 18 May 2020 \\ Accepted: 24 Sept 2020 \\ KEYWORDS \\ Segmentation; \\ Parametric Kernel Graph \\ Cut; Microcalcification; \\ Abnormalities
}

\section{INTRODUCTION}

Breast cancer is the most prevalent cancer, ranking second worldwide and becoming the leading mortality cause among women [1]. The survival rate is enormously enhanced if breast abnormalities are detected at an early stage. Among the screening modalities, mammography is widely used as a gold standard in early detection of breast cancer [2]. The mammography images make it probable to abnormalities in the breast such as microcalcification.

Microcalcification is a tiny deposit of calcium that has hoarded in the breast tissue tends to make the suspected region not seen through complementary views on the mammograms. Moreover, radiologists only diagnose them visually which may lead to human errors, detection errors, and thus this can lead to late detection. The mammogram image containing microcalcification must undergo segmentation process where the image can be transformed into more significant for evaluation purposes.

Segmentation is a process of divided an image into meaningful regions which correspond to the same criteria [3]. More precisely, image segmentation can be defined as a process of allocating each pixel in an image with the same features such as color, intensity, or texture in same visual characteristic. Generally, image segmentation approaches can be divided into two categories, which are edge-based and region-based. Edge-based approach is based on intensity discontinuity and linked to form the boundaries of the region [4]. While, region-based approach will segment the image into regions that having similar sets of pixels [5].

However, the drawbacks of edge-based approaches are sensitive to noise, not robust in practice and sometimes it cannot overcome the difficulties in extracting the boundaries of interested region [6]. Thus, this study used region-based method in segmenting microcalcification. The Graph Cut algorithm is one of the region-based method which capable in solving a wide range of computer visions, including image segmentation by maintaining a global minimum energy function, where the basis of graph theory was implemented [7]. With this criteria, the Graph Cut algorithm has become more robust in practices, efficient and can overcome the 2D and 3D problems [8].

Unsupervised Graph Cut algorithm which does not require user interaction needs to use a piecewise model or Gaussian generalization so that it can recognize the data term. Unfortunately, these models are not flexible as different images may require different models [9]. Even every region in the same image may also require different model. Therefore, an improvisation of Graph Cut method, which is Parametric Kernel Graph Cut, was proposed by [10] for multiple region segmentation. Their study investigates kernel mapping to bring the unsupervised Graph Cut algorithm become more general that can segment various images without an assumption regarding the image model.

Hence, this paper aims to focus on performing the Parametric Kernel Graph Cut algorithm in microcalcification segmentation. By the end of this study, the performance of the proposed method will be evaluated by using two quantitative measures: Dice and Jaccard coefficient and the accuracy and sensitivity by using percentage relative error of area between method and experts.

The remainder of this paper is organized as follows: Section 2 briefly presents an overview of Parametric Kernel Graph Cut Algorithm. Section 3 explains the implementation of the proposed method which comprises of four main phases. Section 4 provides the experimental results and parameter analysis about the method. Section 5 concludes the paper. 


\section{PARAMETRIC KERNEL GRAPH CUT ALGORITHM}

Graph Cut method was originally introduced by [10]. Graph Cut used a weighted directed graph $G=\langle V, E\rangle$ where $V$ is a set of vertices and $E$ is a set of directed edges connecting the neighboring vertices. There are two special designated nodes or can be called as terminal nodes that will represent the foreground (object) and background, which are the source $s$ and the sink $b$. Therefore, $G=P \cup\langle s, b\rangle$. Besides that, the other components in Graph Cut are two types of links, which are defined as $t$-links and $n$-links. The $t$-links are edges between the pixels and terminal nodes while $n$-links are the edges between the pixels. For better illustrations, Figure 1 shows the simple 2D segmentation example of graph $\mathrm{G}$ for a 3 x 3 image [10].
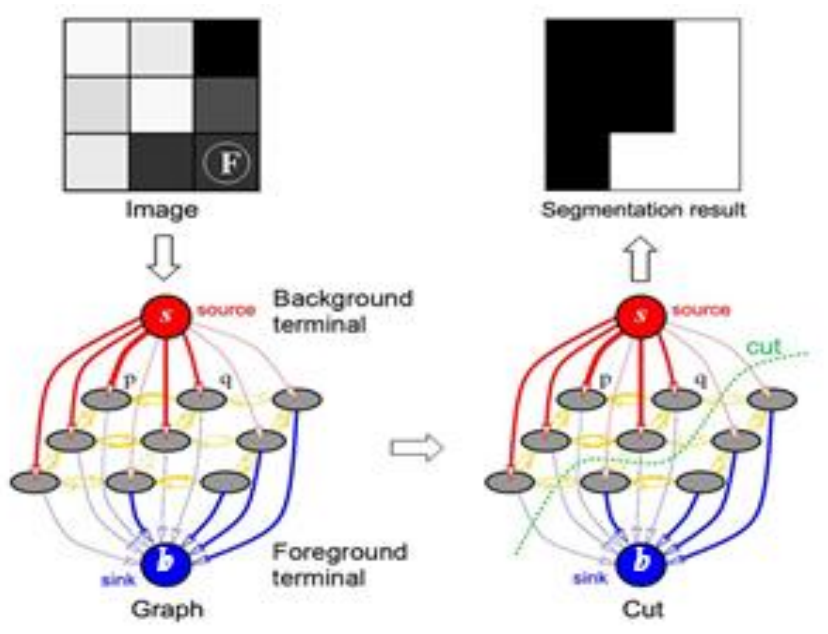

Figure 1. A simple 2D segmentation example of graph $G$ for a 3 x 3 image

Figure 1 illustrates a simple 2D segmentation example of graph $G$ for a 3 x 3 image. From Figure 1, yellow edges represent the $n$-links while red and blue edges represent $t$-links. Grey vertices represent all pixels in the graph while red and blue vertices represent source $s$ and $\operatorname{sink} b$, respectively. Each pixel has both $t$-links connecting it to each terminal $s$ and $b$. The $s-b$ cut is a subset of edges $C \subset E$ when the terminal $s$ and $b$ became separable on the induced graph $G(C)=\langle V, E \backslash C\rangle$. Green line in Figure 1 shows the illustrations of $s$ - $b$ cut on graph $\mathrm{G}$.

Any solution of cut $\mathrm{C}$ must be feasible on graph $\mathrm{G}$ such that:

- C severs exactly one $t$-links for each pixel

- $\{p, q\} \in C$ if $p$ and $q$ are linked to different terminals

Graph Cut theorem relates two quantities which is max flow-min cut theorem. Note that the cut is minimal in the sense that none of its subsets separates the terminals into the same two subgraphs. The maximum flow from the source s to sink $\mathrm{b}$ is equivalent to the net flow of the edges in the minimum cut. Therefore, it means that min-cut problem can be solved directly by solving the max-flow problem [11].

Graph Cut algorithm, can achieve an optimum solution when the energy optimization is equivalent to the minimum cut. A minimum cut is a cut whose capacity is the least over all the $s$ - $b$ cuts of the network. Since a cut will separate both terminals, each pixel cannot sever both t-links. For any feasible cut, there exist a unique corresponding segmentation $A(C)$ which can be defined as,

$$
A_{p}(C)= \begin{cases}\text { " object" } & \text { if }\{p, s\} \in C \\ \text { "background } " & \text { if }\{p, b\} \in C\end{cases}
$$

Based on the Equation (1), let $A=\left(A_{1}, A_{2}, \ldots, A_{p}\right)$, where $A$ defines as a segmentation vector and for each components in which can be either "object" or "background". The segmentation functional for energy optimisation of Graph Cut can be written as the following formula,

$$
E(A)=R(A)+\alpha B(A)
$$

where, $R(A)$ is the regional term which comprises the region properties. In other words, $R(A)$ act as penalties in assigning the pixel as a background or object. $B(A)$ is a boundary term that assumes the smoothness term of the boundary properties, whereby it will determine the discontinuity term between the pixels. The coefficient $\alpha$ represents as a positive factor that will control the smoothness term in labelling. Specifically, Equation (2) is written as, 


$$
E\left(\left\{\mu_{l}\right\}, A\right)=\sum_{l \in L} \sum_{p \in R_{l}}\left(\mu_{l}-I_{p}\right)^{2}+\alpha \sum_{\{p, q\} \in N} B_{\{p, q\}} \delta\left(A_{p}, A_{q}\right)
$$

$L$ refers to the set of the region label which is background label or object label of the pixel $p$ while $\mu_{l}$ refers to the value of the label. $N$ is a set of neighbourhood pixels.

However, Equation (3) is the segmentation functional for the original Graph Cut. For Parametric Kernel Graph Cut Algorithm (PKGC), kernel function will be substituted into the regional term, $R(A)$. In this paper, the proposed kernel trick is able to solve a nonlinear problem into a simple linear problem, so that the image data will have a better separability. The substitution of the kernel function gives,

$$
E_{K}\left(\left\{\mu_{l}\right\}, A\right)=\sum_{l \in L} \sum_{p \in R_{l}} R_{K}\left(I_{p}, \mu_{l}\right)+\alpha \sum_{\{p, q\} \in N} B_{\{p, q\}} \delta\left(A_{p}, A_{q}\right)
$$

where $R_{K}\left(I_{p}, \mu_{l}\right)$ is the result of the substitution of the kernel function, where the formulation is derived by [10] which derived to,

$$
R_{K}\left(I_{p}, \mu_{l}\right)=K\left(I_{p}, I_{p}\right)+K\left(\mu_{l}, \mu_{l}\right)-2 K\left(I_{p}, \mu_{l}\right)
$$

$I_{p}$ represents the intensity value of the pixel $p$ while $\mu_{l}$ value will be based on which label that pixel $p$ is belong to. For the Equation (5), this paper chose RBF kernel in clustering the data since [12] state that RBF kernel generated more accurate result as compared to the other kernel function where the function is,

$$
K(x, y)=\exp \left(\frac{-\|x-y\|^{2}}{2 \sigma^{2}}\right)
$$

On the other hand, for the boundary term $B(A), \delta\left(A_{p}, A_{q}\right)$ value in Equation (4) is equal to 1 when both $p$ and $q$ are in different group partitions and 0 when $p$ and $q$ are in the same group partitions while $B_{\{p, q\}}$ uses the Gaussian probability distribution which is multiplied by the inverse Euclidean distance and written as,

$$
B_{\{p, q\}}=\exp \left(-\frac{\left(I_{p}-I_{q}\right)^{2}}{2 \sigma^{2}}\right) \cdot \frac{1}{\operatorname{dist}(p, q)}
$$

The larger the $B_{\{p, q\}}$, the higher the similarity between the intensity of pixels $p$ and $q . \operatorname{dist}(p, q)$ is the Euclidean distance between $p$ and $q$ while $\sigma$ is a constant where the value should be less than the $\operatorname{dist}(p, q) . I_{p}$ and $I_{q}$ are the intensities of pixel $p$ and $q$, respectively. The value that will be returned from the boundary term is only within 0 to 1 , whereby the higher the value means the higher the similarity between intensity value for pixel $p$ and $q$.

For every graph, each edges $e \in E$ is assigned as non-negative weight (cost), $w(e)$ and the total weight of a graph refers to the total cost of a cut which can be defined as,

$$
|C|=\sum_{e \in C} w(e)=\sum_{(p, q) \in \Omega} w(e=(p, q)) d_{e=(p, q)}
$$

where, $w(e)$ is the cost of the edges and $d_{e=(p, q)}$ is equal to 1 when $p$ and $q$ are in different groups and 0 if $p$ and $q$ are in the same groups. As mentioned earlier, there are two types of edges, $n$-links and $t$-links. Let $\{p, q\}$ be a pair of a neighboring pixels. Each pixel has both $t$-links connecting it to each terminal $s$ and $b,\{p, s\}$ and $\{p, b\}$.

Parametric Kernel Graph Cut Algorithm generates the optimal segmentation result in terms of properties that are built into the edge weights. Therefore, the segmentation result that generated is optimal since the cost of the cut are built based on the properties for each edge. The following Table 1 gives the exact weights of all these edges [10]. 
Table 1. The weight of edges

\begin{tabular}{|c|c|c|}
\hline Edge & Weight (cost) & For \\
\hline$\{p, q\}(n-$ link $)$ & $B_{\{p, q\}}$ & $\{p, q\} \in N$ \\
& 0 & $\{p, q\} \notin N$ \\
\hline$\{p, s\}(t-$ link $)$ & $R_{K}\left(I_{p}, \mu_{s}\right)+\sum_{q \in N_{p}} B_{\{s, q\}} \delta\left(\mu_{s}, A_{q}\right)$ & $\forall p \in V$ \\
\hline$\{p, b\}(t-$ link $)$ & $R_{K}\left(I_{p}, \mu_{b}\right)+\sum_{q \in N_{p}} B_{\{b, q\}} \delta\left(\mu_{b}, A_{q}\right)$ & $\forall p \in V$ \\
\hline
\end{tabular}

Based on Table 1, the weight of edges for both $n$-link and t-link. The weight for each edge is assigned based on each partition and all the weights are referred as the minimum cut for each edge. Then, the boundary will be drawn based on the specified weights in Table 1. Therefore, a graph will be constructed according to the obtained optimized solution of the Equation (4) via max flow-min cut algorithm.

\section{IMPLEMENTATION}

The implementation for this paper begins with data acquisition. 25 mammogram images were provided from the National Cancer Society Malaysia (NCSM) and all the microcalcification were already confirmed by the radiologist. At the second phase, the image data was clustered into $k$ regions by using K-means clustering. Since there were two clusters that have been set up to, thus the image is segmented according to these regions at the next phase where this paper focused on multiregional segmentation of mammograms image by using Parametric Kernel Graph Cut Algorithm. The outcome of the segmentation was then proceeded with the performance evaluation. This paper was focused on the accuracy and sensitivity so that the capability of the method can be tested by applying two evaluation techniques, which were Dice and Jaccard coefficient and also based on the accuracy and sensitivity using percentage relative error of area between the proposed method and expert. The flows of implementation phases are shown in Figure 2

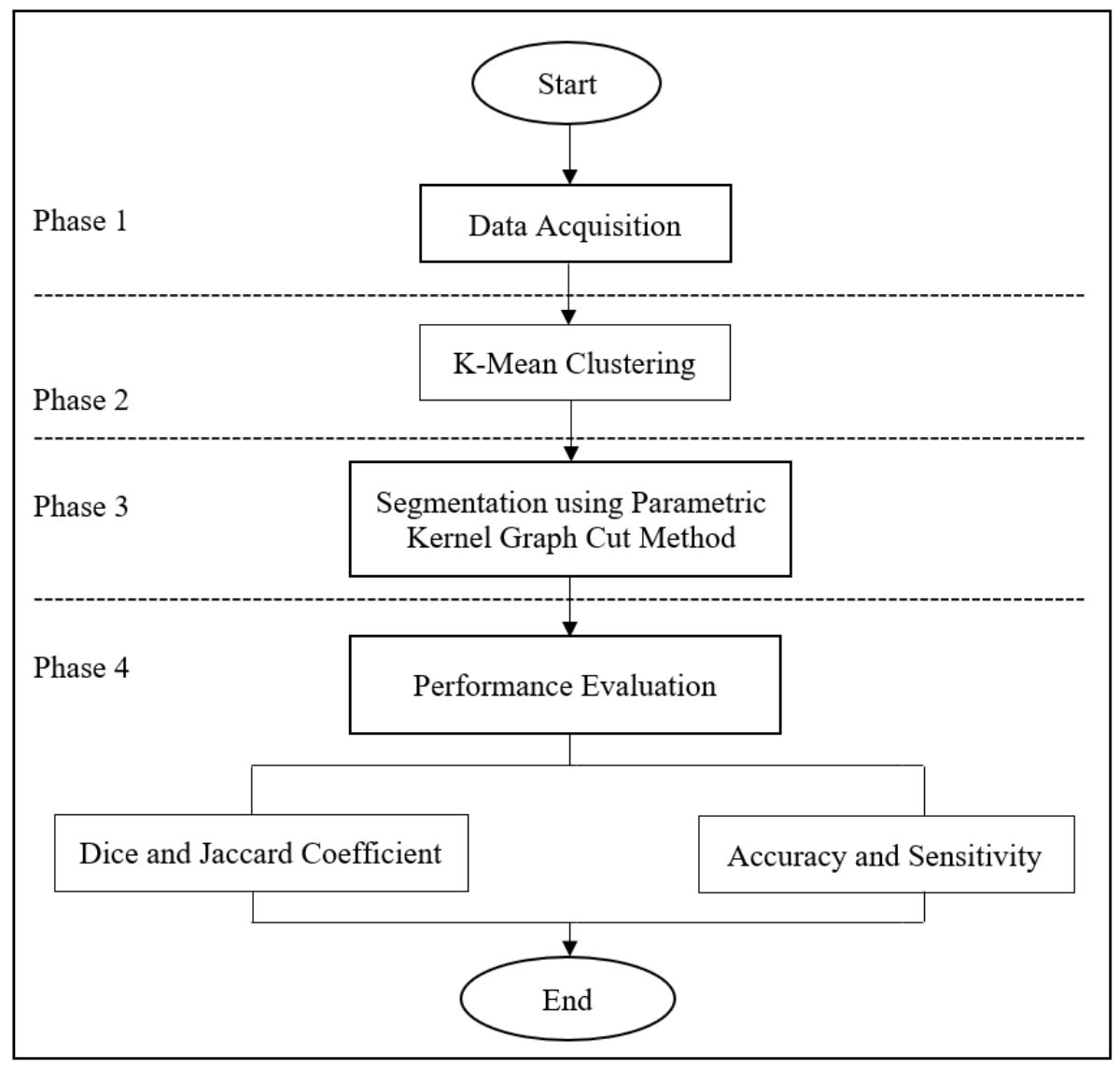

Figure 2. The Implementation Phases 


\section{Phase 1: Data Acquisition}

The images of the microcalcification are set in standard size which is in $200 \mathrm{x} 200$ pixels in the format of Portable Network Graphics (PNG). The software that has been used for this study is MATLAB R2014a. The mammogram image is stored as an 8-bit integer (2D image) that has a specific range of values from 0 to 255 where 0 value indicates as a black and 255 value indicates as a white. These values will differentiate the level of intensity for each pixel.

\section{Phase 2: K-Means Clustering}

As for the second phase, the image data are clustered into $k$ regions using the K-means clustering algorithm. The main objective of cluster analysis is to divide the image data set into many disjoint groups or clusters [13]. The ' $\mathrm{K}$ ' in $\mathrm{K}$-means denotes the number of clusters that will be created. Clustering is like finding the similarity of the object such that the object is homogeneous within the group and heterogeneous between the groups where the similarity of the data set is measured based on the Euclidean distance. The following steps explained the processes involved in K-means clustering algorithm.

Step 1: Set the number of clusters into $k$ regions. The data need to be clustered into $k$ regions. In this study, the number of clusters are assigned to 2 (let $k=2$ ). This is because the number of clusters are representing both background and an object which is the microcalcification that will be extracted.

Step 2: Initialize the centroids. Centroids or centres were chosen based on the number of clusters. The centroids can either be randomly selected or sort the data into $k$, then choose one data point from each cluster. Since this study has 2 clusters. So, two points were randomly selected as cluster centroids.

Step 3: Calculate the Euclidean distance. Since the centroids were already assigned at the previous step then the Euclidean distances were computed between the centroids and all the objects. The distances were calculated using following formula

$$
d\left(c_{k}, \text { object }_{n}\right)=\sqrt{\left(c_{k}-\text { object }_{n}\right)^{2}}
$$

where $k$ is the number of clusters and $n$ is the number of objects.

Step 4: Assign each object to the closest cluster. Since there are two clusters, so each object will be assigning into one of the clusters.

Step 5: Update the cluster centroids. After all the objects were already assigned to each cluster, calculate the new centroid for each cluster. To find the new centroids, calculate the average of all data points in each cluster.

Step 6: Repeat step 3, 4 and 5 until the same point are assigned to each cluster in consecutive rounds. Since there are two clusters that has been set up, therefore the image is segmented according to these regions where each pixel is assigned to its cluster and Parametric Graph Cut algorithm will poses smoothness constraint at the next phase.

\section{Phase 3: Segmentation using Parametric Kernel Graph Cut Algorithm}

In this phase, Parametric Kernel Graph Cut is used to segment the microcalcification in mammogram images. The steps are described as follows:

Step 1: Find the regional term. Regional term comprises the smoothness term which refers to the region properties of a graph. In other words, the term act as a penalty in assigning the pixel as a background or object. The region properties will assume the penalties for assigning each pixel to object or background [14]. The equation of the regional term is computes as in Equation (5).

Step 2: Calculate the boundary term. Boundary term, $B(A)$ refers to the data cost which comprises the boundary properties of a graph. In Graph Cut, it is also known as the boundary penalties for the discontinuity between a pair of pixels (Wang et al., 2013). The equation for boundary term is

$$
B(A)=\sum_{\{p, q\} \in N} B_{\{p, q\}} \delta\left(A_{p}, A_{q}\right)
$$

where $B_{\{p, q\}}$ can be obtained through Equation (7) and $N$ is a set of all neighboring pixels. Boundary term $B(A)$ act as the penalties in boundary properties for the discontinuity term between the pixels. The larger the $B_{\{p, q\}}$, the higher the similarity between the intensity of pixels $p$ and $q$. While $\delta\left(A_{p}, A_{q}\right)$ is a non-zero when both $p$ and $q$ are in different group partitions. The value for $\sigma$ set as a constant value which is $0.5 . B_{\{p, q\}}$ uses Gaussian probability distribution multiplied by the inverse Euclidean distance. 
Step 3: Find the energy term. The energy term refers to the energy segmentation functional of Graph Cut which can be written as in Equation (2).

Step 4: Find the minimum cut. The goal of Parametric Kernel Graph Cut algorithm is to compute the best cut such that the cuts would provide the optimal segmentation. Optimal segmentation can be achieved by finding the minimum cost cuts, which refers to the sum of its edge weight as in equation (8). The minimum cost for a cut of a graph can be obtained by finding all three edges that listed in Table 1 . From the table, the first edge refers to $n$-links which means the edges of all the neighboring pixels. Note that any solution of cut $\mathrm{C}$ must be feasible on graph $\mathrm{G}$ such that

- C severs exactly one $\mathrm{t}$-links for each pixel

- $\{p, q\} \in C$ if $\mathrm{p}$ and $\mathrm{q}$ are linked to different terminals

- $A_{p}=F$ if $\{p, b\} \in C$

- $A_{p}=O$ if $\{p, s\} \in C$

Since a cut will separate both terminals for object and background, a minimum cut is severing when both pixel p and $\mathrm{q}$ are connected to the opposite terminal and that is why the neighboring pixels should be in the different groups.

\section{Phase 4: Performance Evaluation of Segmentation Results}

The results were then evaluated by applying two quantitative measures: the evaluation of Dice and Jaccard coefficient, accuracy and sensitivity based on percentage relative error of area between the method and experts.

\section{Dice and Jaccard Coefficient}

Dice and Jaccard coefficient is an overlapping measure that is often utilized to quantify the similarity between two sample sets [15]. In this paper, the evaluation of the performance was determined by the overlapping accuracy ratio of segmented image which was generated by PKGC against the ground truth image, which is the image that was marked from the radiologist. Both images need to be converted to binary images. In order to calculate Dice coefficient, the following equation is used [16].

$$
\text { Dice }=\frac{2 \times T P}{(2 \times T P+F P+F N)}
$$

where $T P$ is the number of true positives when both image have the same pixel value of $1, F P$ is the number of false positives where the pixel value of 1 only in the PKGC. FN is false negatives which refer to the number of pixels with value 1 that only in the ground truth image. Then, as for the Jaccard coefficient is calculated by using the following formula.

$$
\text { Jaccard }=\frac{\text { Dice }}{2-\text { Dice }}
$$

The result was ranged from 0 to 1 , where the 0 value indicated no overlap occurrence and 1 indicated the complete congruence. Therefore, the higher ratio will depict better results.

\section{Accuracy}

Accuracy is defined as a measurement on how close a result compared with a true value. In this case, the accuracy was determined by the closeness of the PKGC segmentation result and radiologist result. When the results of image segmentation by using PKGC were obtained, the average area of the segmented images was calculated as well as the average areas of the expert findings.

\section{Sensitivity}

The sensitivity is the proportion of true positives that are correctly identified by the test [17]. Higher sensitivity values give the best result. The sensitivity is evaluated based on the recognition statistic from [18] as in Table 2.

Table 2: Recognition Statistic of Segmentation Result

\begin{tabular}{lc}
\hline Recognition Statistic & Range of Relative Error (\%) \\
\hline VERY GOOD & {$[0,2)$} \\
\hline GOOD & {$[2,4)$} \\
\hline AVERAGE & {$[4,6)$} \\
\hline BELOW AVERAGE & {$[6,8)$} \\
\hline POOR & $\geq 8$ \\
\hline
\end{tabular}

Based on Table 2, the percentage relative error obtained from each image is categorized into 5 categories ranging from very good to poor cases. True positive (TP) is considered from very good to average cases. Then, sensitivity is measured based on total number of TP divide by the total number of images. 


\section{EXPERIMENTAL RESULTS}

Parametric Kernel Graph Cut Algorithm has successfully segmented all 25 mammogram images. Figure 3 shows samples of successful segmentation results.
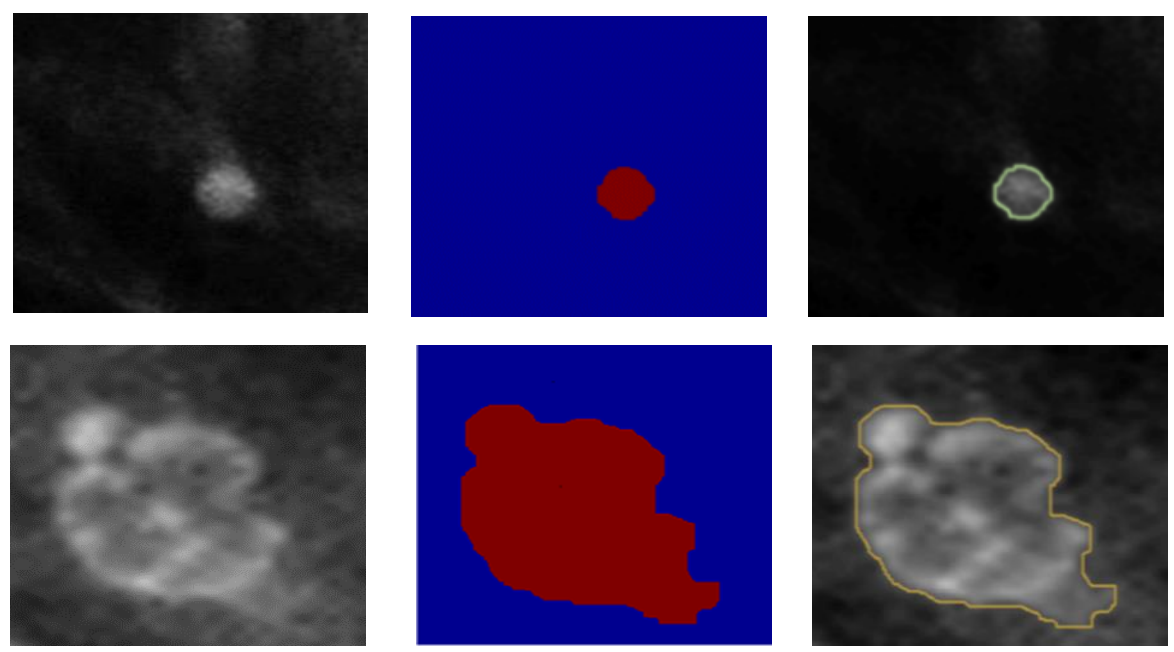

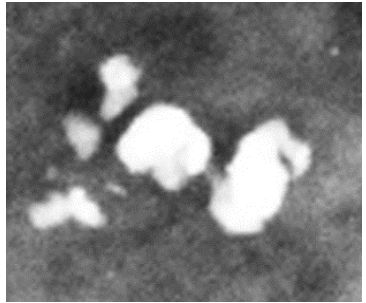

(a) Original images

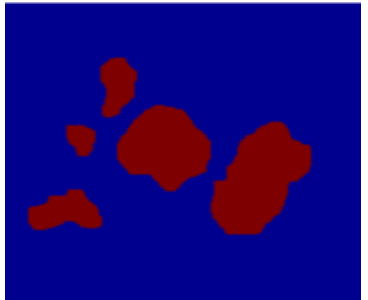

(b) Region of segmentation results

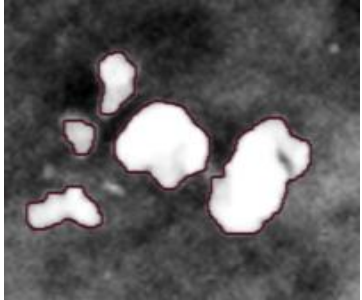

(c) Boundary of segmentation results

Figure 3. Segmentation Results by Using PKGC

Figure 3 shows three samples of mammogram images which were successfully segmented by using PKGC. PKGC had successfully produces both region and boundary of segmented image. The result partitioned the image into two regions, which were object and background and assigned each of them with two different kinds of color as depicted in Figure 3(b). Figure 3(c) depicts the boundary of the region which shows the actual shape of the microcalcification so that this shape can help radiologist in analyzing whether these tumors are benign or malignant. Such a result enables to help the radiologist to calculate the microcalcification area, according to the boundary circumference. Moreover, the different curve lines of boundary play a crucial role in determining whether the microcalcification is a cancerous cell or not.

One of the objectives of kernel functions in PKGC is to ensure that the Graph Cut formulation has the ability to segment a multiregion image [10]. The third image in Figure 3 proves this ability, whereby PKGC was capable in segmenting the clusters of microcalcification. The intensity grey level of the second image was quite blurred as compared to the other images. Since the segmentation of the region-based method determined by the similarity, color and texture of the image, the segmentation might be quite challenging in detecting the microcalcification. However, by using PKGC, the precise boundary was properly segmented. The robustness of PKGC improve the flexibility of the method in segmenting various kinds of image.

\section{Parameter Analysis of Parametric Kernel Graph Cut Algorithn}

Based on the Equation (2), $\alpha$ is a variable that refers to the weight of image smoothness. It is also known as the degree of opacity of the pixel and the value can be specified from 0 to 255 . This is because the $\alpha$ value represents the transparency information of the pixel, whereby the 0 and 255 values represent the full transparency and the full opacity, respectively. Obviously, the boundaries of each image are varied in size and shape. Also, there is intensity inhomogeneity for every pixel. Therefore, this parameter needs to be customised beforehand to achieve precise and successful results in image segmentation.

During the segmentation process, several values of $\alpha$ were tested until the optimum segmentation was achieved. The range value which was set up for all the mammogram images was between 0 until 4.5 . In this paper, most of the mammogram images was set up with 0.1 as the value of $\alpha$. The value depends on the intensity level of the image. For example, any blurred image will be set up in low values of $\alpha$ since a low transparency was needed to segment this kind of image successfully. Meanwhile, any solid and clear image required a higher $\alpha$. Figure 5 shows the results of three different setting of $\alpha$ parameter. 

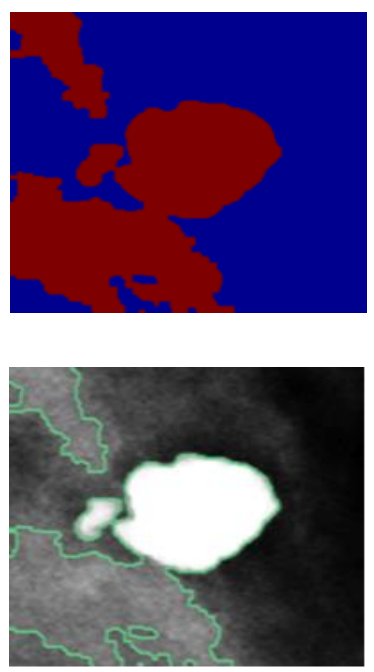

(a) Low value $(\alpha=0.1)$
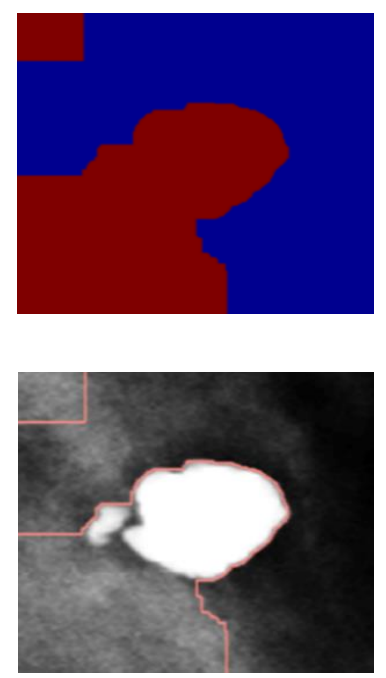

(b) High value $(\alpha=2.0)$
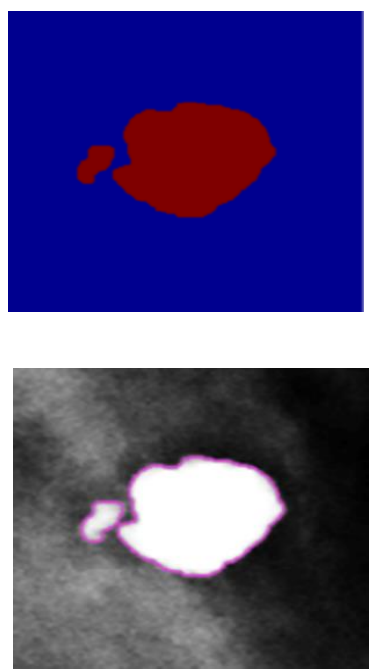

(c) Optimum value $(\alpha=0.5)$

Figure 4. Segmentation Results with Different $\alpha$ Values

Referring on Figure 4, the segmentation results are based on multiple setting of $\alpha$ values. The $\alpha$ values which are too low or too high may lead to a wrong segmentation of the region. In this experiment, both too low and too high values of parameter were set up to see the differences between the results and all the results did not give an accurate segmentation. The parameter needed to be customised beforehand to achieve precise and successful results in the image segmentation, which means that the parameter needed to be set up based on the suitability and characteristic of the mammogram image. Figure 4(c) presented the image of segmentation result in which the parameters were set such that the segmentation result was most accurate, where 0.5 was the optimum value of $\alpha$ for the image.

Based on the Equation (4), there were three parameters which are $\sigma, \mathrm{x}$ and $\mathrm{y}$ and all of them were related to each other in order to enable the function to segment the images. Sigma plays an important role to be an amplifier of the distance between $\mathrm{x}$ and $\mathrm{y}$. In this study all these parameters were set by default. If the distance between $\mathrm{x}$ and $\mathrm{y}$ is much larger than sigma, the kernel function tends to be zero. Figure 5 illustrates the experiments of the segmentation results by using different $\sigma$ values.
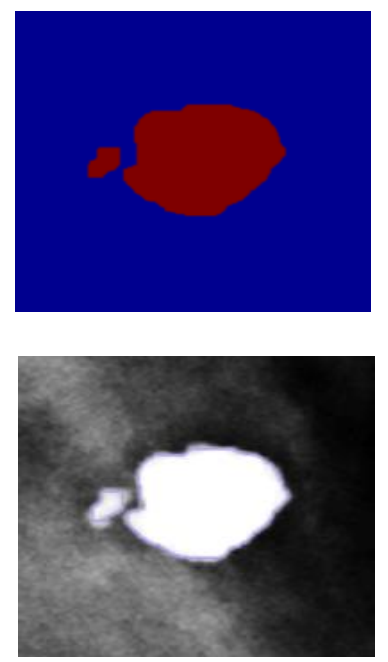

(a) Low value of $(\sigma=0.1)$
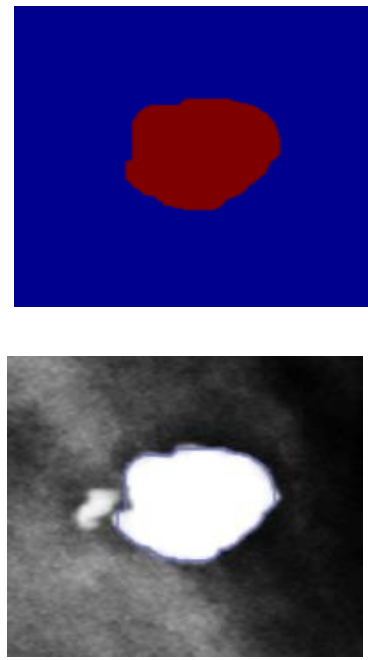

(b) High value $(\sigma=3.0)$
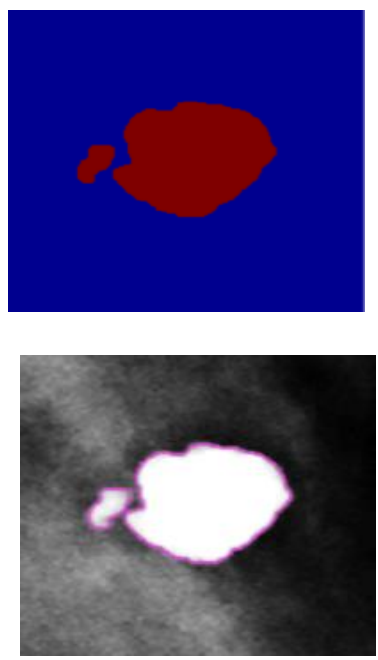

(c) Optimum value $(\sigma=0.5)$

Figure 5. Segmentation Results with Different $\sigma$ Values

Figure 5 show the segmentation results by using different $\sigma$ values. The values of $\sigma$ may lead to a different segmentation of the region. The larger the sigma is, the higher the possibilities in making wrong classification since larger sigma tends to make a much more general classifier (Figure 5(b)) while smaller sigma tends to make a local classifier. For smaller sigma, the decision boundary tends to be strict and sharp as shown in Figure 5(a). This parameter was set by default for all images with 0.5 since it is the optimum values of $\sigma$. 


\section{Performances Evaluation}

Figure 6 shows the result for all images of Dice and Jaccard coefficient.

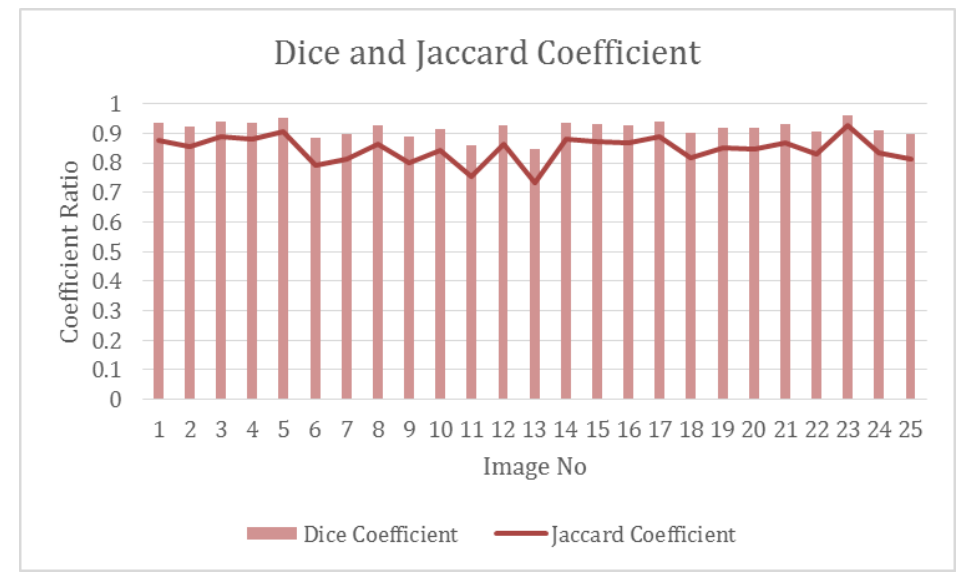

Figure 6. The Result of Dice and Jaccard Coefficient

Figure 6 illustrates the results for all mammogram images that were obtained from Dice and Jaccard coefficient. All the ratio results can be considered satisfying enough for the overlapping ratio comparison where all the images produce the average of $91.67 \%$ for Dice coefficient and $84.72 \%$ for Jaccard coefficient. Apart from that, the results for Dice coefficient were higher as compared to the Jaccard Coefficient. This is due to the fact that Jaccard is more sensitive, whereby Jaccard is numerically more sensitive to mismatch, especially when there is no reasonably strong overlap segmentation [19].

The results of percentage relative error of areas for all 25 images are shown in Figure 7.

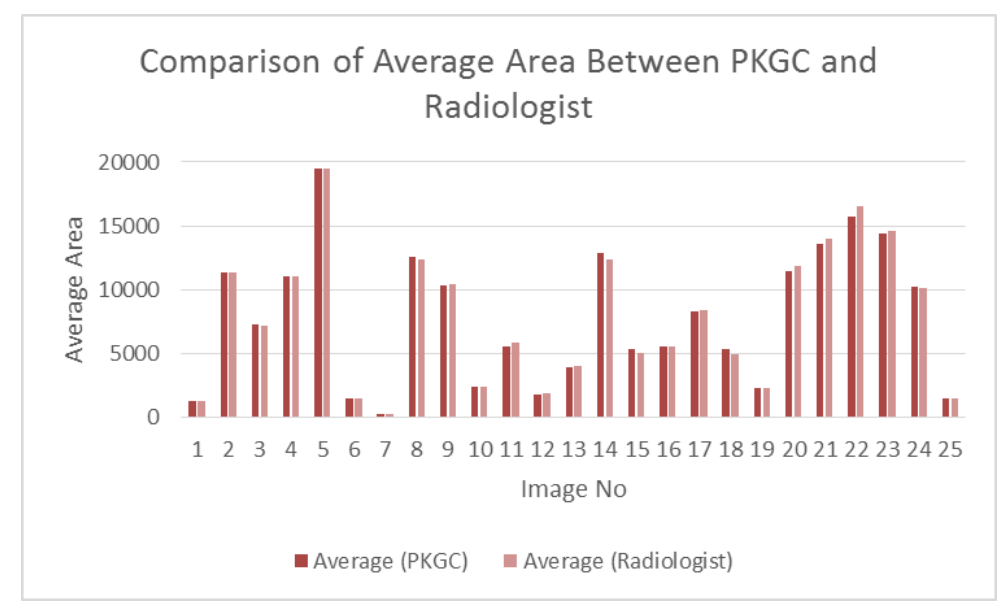

Figure 7. Bar Chart of Average Area for PKGC and Radiologist

Based on Figure 7, the comparison of all the average area was illustrated in the form of bar chart for better visualization. There was only a small difference between the area that obtained by PKGC and radiologists. The accuracy that generated was $97.84 \%$ where it has only less than $3 \%$ of the inaccurate result. It can be concluded that the area that is obtained by using PKGC can be considered as accurately segmented.

Next, in term of sensitivity, the frequency of percentage relative error for each images were categorized into 5 categories based on Table 3, ranging from very good to poor cases. From very good to average cases, the true positive (TP) was considered.

Table 3. The Frequency of Percentage Relative Error

\begin{tabular}{lcc}
\hline Recognition Statistic & Number of Image & Range of Relative Error (\%) \\
\hline VERY GOOD & 16 & {$[0,2)$} \\
GOOD & 4 & {$[2,4)$} \\
AVERAGE & 4 & {$[4,6)$} \\
\hline BELOW AVERAGE & 0 & {$[6,8)$} \\
POOR & 1 & $\geq 8$ \\
\hline Sensitivity (\%) & & $96 \%$ \\
\hline
\end{tabular}


Based on Table 3, all images were categorised based on the relative error for each image. PKGC obtained 96\% sensitivity. Out of 25 images, PKGC had successfully obtained 24 images for true positive category while only one image was categorised as poor cases. For better illustration, the result was visualised in the form of pie chart, as shown in the following Figure 8.

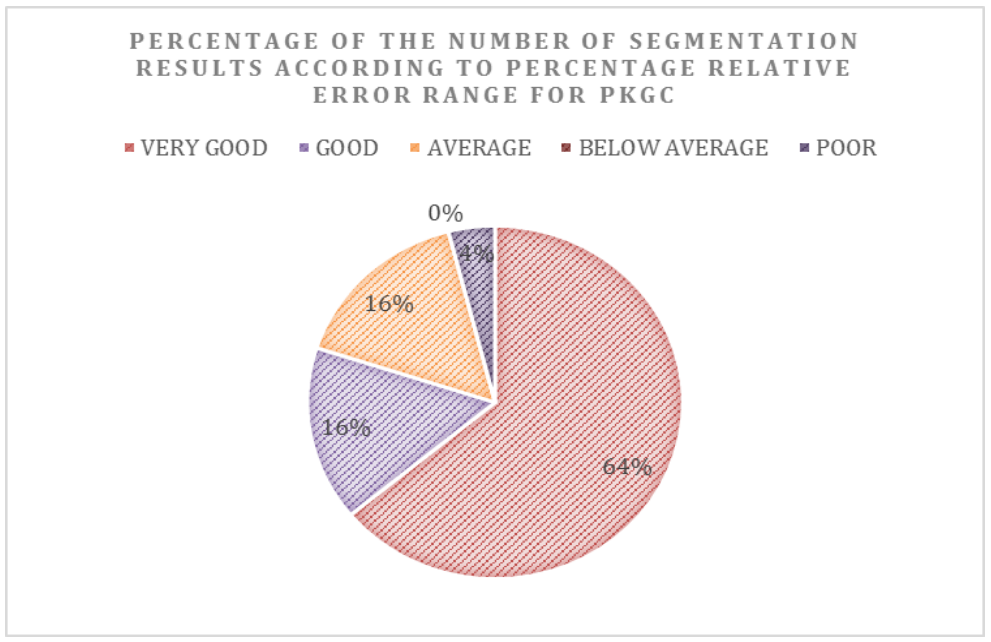

Figure 8. A Pie Chart of Recognition Statistic in PKGC

Figure 8 illustrates the percentages result for the number of images obtained based on the percentage relative error range for all five categories. The category for very good cases obtained $64 \%$ and it was the highest among all of the categories. Besides, only $4 \%$ was for poor cases category, whereby only one image was diagnosed as a poor case. Therefore, it can be concluded that the segmentation result of PKGC gave optimum result where it gave $96 \%$ for true positive category.

\section{CONCLUSION}

In the nutshell, the main purpose of this paper was to segment the microcalcification on mammogram images by using Parametric Kernel Graph Cut Algorithm. The proposed method was the improvisation of Graph Cut method where it used kernel mapping in the segmentation function instead of using piecewise model or Gaussian generalization where these models are not flexible enough since different image may require different models. Hence, this paper aims to directly segment the mammogram images without using any models.

The objective of the improvisation is to investigate the kernel mapping to bring the unsupervised Graph Cut formulation become more general especially on multiregional segmentation. The flexibility and robustness of Parametric Kernel Graph Cut was proved, where the method could segment the microcalcification in the mammogram images and achieve outstanding results in the evaluation performances. Therefore, Parametric Kernel Graph Cut Algorithm proved its ability in segmenting the microcalcification robustly and efficiently.

The future plan for this research aims to implement this method to the other type of images as well as the other type of abnormality. Also, since the parameters in this proposed method were set manually, thus other methods will be explored in such a way that all the parameters can be generated automatically.

\section{ACKNOWLEDGEMENT}

This research was supported by Universiti Teknologi MARA (UiTM), Cawangan Negeri Sembilan, Seremban Campus. The authors would like to acknowledge UiTM for the support and contribution. The author(s) also would like to acknowledge Prof Dr Rozi Mahmud (Expert Radiologist) for the support and involvement in this study.

\section{REFERENCES}

[1] World Health Organization. "Cancer in Malaysia." Globocan. https://gco.iarc.fr/today/data/factsheets/cancers/20-Breastfact-sheet.pdf (accessed 2020).

[2] N. Al-Najdawi, M. Biltawi, and S. Tedmori, "Mammogram image visual enhancement, mass segmentation and classification," Applied Soft Computing, vol. 35, pp. 175-185, 2015/10/01/ 2015.

[3] D. Kaur and Y. Kaur, "Various Image Segmentation Techniques: A Review," 2014.

[4] N. M. Zaitoun and M. J. Aqel, "Survey on Image Segmentation Techniques," Procedia Computer Science, vol. 65, pp. 797806, 2015/01/01/ 2015.

[5] R. C. Gonzalez and R. E. Woods, Digital Image Processing (3rd Edition). Prentice-Hall, Inc., 2006.

[6] S. Don, E. Choi, and D. Min, Breast Mass Segmentation in Digital Mammography Using Graph Cuts. 2011, pp. 88-96.

[7] Y. Boykov and G. Funka-Lea, "Graph Cuts and Efficient N-D Image Segmentation," International Journal of Computer Vision, vol. 70, no. 2, pp. 109-131, 2006/11/01 2006. 
[8] V. Kolmogorov and Y. Boykov, "What metrics can be approximated by geo-cuts, or global optimization of length/area and flux," in Tenth IEEE International Conference on Computer Vision (ICCV'05) Volume 1, 17-21 Oct. 2005 2005, vol. 1, pp. 564-571 Vol. 1.

[9] N. Vu and B. Manjunath, Shape prior segmentation of multiple objects with graph cuts. 2008, pp. 1-8.

[10] M. Ben Salah, A. Mitiche, and I. Ben Ayed, "Multiregion Image Segmentation by Parametric Kernel Graph Cuts," Image Processing, IEEE Transactions on, vol. 20, pp. 545-557, 03/01 2011.

[11] M. Kulkarni, "Image and video segmentation using graph cuts," University of Cape Town, 2010. [Online]. Available: http://hdl.handle.net/11427/5190

[12] W. Chen, "New RBF Collocation Methods and Kernel RBF with Applications," in Meshfree Methods for Partial Differential Equations, Berlin, Heidelberg, M. Griebel and M. A. Schweitzer, Eds., 2003// 2003: Springer Berlin Heidelberg, pp. 75-86.

[13] L. Miao, "Comparative Analysis of Two Clustering Algorithms: K-means and FSDP (Fast Search and Find of Density Peaks)," 2015.

[14] H. Wang, H. Zhang, and N. Ray, "Adaptive shape prior in graph cut image segmentation," Pattern Recognition, vol. 46, no. 5, pp. 1409-1414, 2013/05/01/2013.

[15] E. Tiu. "Metrics to Evaluate your Semantic Segmentation Model." https://towardsdatascience.com/metrics-to-evaluate-yoursemantic-segmentation-model-6bcb99639aa2 (accessed.

[16] M. P. Sampat, Z. Wang, S. Gupta, A. C. Bovik, and M. K. Markey, "Complex Wavelet Structural Similarity: A New Image Similarity Index," IEEE Transactions on Image Processing, vol. 18, no. 11, pp. 2385-2401, 2009.

[17] D. G. Altman and J. M. Bland, "Diagnostic tests. 1: Sensitivity and specificity," BMJ: British Medical Journal, vol. 308 , no. 6943, p. 1552, 1994.

[18] W. Zhu, N. Zeng, and N. Wang, "Sensitivity, specificity, accuracy, associated confidence interval and ROC analysis with practical SAS implementations," NESUG proceedings: health care and life sciences, Baltimore, Maryland, vol. 19, p. 67, 2010.

[19] W. R. Crum, O. Camara, and D. L. Hill, "Generalized overlap measures for evaluation and validation in medical image analysis," IEEE transactions on medical imaging, vol. 25, no. 11, pp. 1451-1461, 2006. 